\title{
Extended bound states and resonances of two fermions on a periodic lattice
}

\author{
A. S. Blaer ${ }^{a}$, H. C. Ren ${ }^{b}$, and O. Tchernyshyov ${ }^{a}$ \\ ${ }^{a}$ Department of Physics, Columbia University, New York, NY 10027 \\ ${ }^{b}$ Department of Physics, Rockefeller University, New York, NY 10021
}

(August 7, 1996)

\begin{abstract}
The high- $T_{c}$ cuprates are possible candidates for $d$-wave superconductivity, with the Cooper pair wave function belonging to a non-trivial irreducible representation of the lattice point group. We argue that this $d$-wave symmetry is related to a special form of the fermionic kinetic energy and does not require any novel pairing mechanism. In this context, we present a detailed study of the bound states and resonances formed by two lattice fermions interacting via a non-retarded potential that is attractive for nearest neighbors but repulsive for other relative positions. In the case of strong binding, a pair formed by fermions on adjacent lattice sites can have a small effective mass, thereby implying a high condensation temperature. For a weakly bound state, a pair with non-trivial symmetry tends to be smaller in size than an $s$-wave pair. These and other findings are discussed in connection with the properties of high- $T_{c}$ cuprate superconductors.
\end{abstract}

PACS numbers: 71.10.Fd, 74.20.-z, 03.65.Ge

\section{INTRODUCTION}

Recently, through angle-resolved photoemission spectroscopy [1] and the tri-crystal Josephson effect [2], a substantial $d$-wave component has been detected in the order parameter of the cuprate superconductors. For tetragonal crystals, such as Tl-based cuprates in which the square symmetry of the $\mathrm{CuO}_{2}$ plane is perfect, the order parameter appears to be entirely $d$-wave. In other superconducting cuprates, with orthorhombic distortions, the order parameter contains a large fraction of $d$-wave. These observations have prompted theorists to look for exotic mechanisms that could lead to a non-trivial symmetry of the order parameter. It may, however, be true that it is simply the fermionic kinetic energy that leads to $d$-wave superconductivity. An exactly solvable problem with two fermions would provide a strong argument for this possibility.

Pines et al. [3] have proposed a one-band model for cuprate superconductors in which quasiparticles hop between copper sites with amplitude $t_{1}<0$ along the sides of square cells and with amplitude $t_{2}>0$ along the diagonals. The interaction between quasiparticles is mediated by magnon exchange and, in the static limit, can be thought of as a repulsive potential for two quasiparticles on the same antiferromagnetic sublattice and as an attractive potential otherwise. In the present paper, we demonstrate that a positive $t_{2}$ is actually a necessary condition for a two-fermion system to have a $d$-wave ground state. In cuprate compounds, a reversed (positive) sign of $t_{2}$ is indeed expected if doped holes occupy $d_{x^{2}-y^{2}}$ orbitals of the copper ions. Such orbitals have four lobes along the principal axes of the $\mathrm{CuO}$ planes, with a positive amplitude along one axis and a negative amplitude along the other. For adjacent copper ions the overlapping lobes have the same sign, while for next-nearest neighbors positive lobes overlap with negative ones. Therefore, a reversed sign of $t_{2}$ occurs.

Measurements of the upper critical magnetic field have revealed a very short coherence length in high- $T_{c}$ cuprates. As we shall see, for a $d$-wave pair, the average distance between the two fermions is on the order of the lattice spacing, even for weak binding. Therefore, these pairs are very localized and may well undergo a Bose-Einstein condensation. The resulting transition temperature $T_{\mathrm{BE}}$ decreases with a growing effective boson mass. For a point-like boson on a lattice (strong on-site binding), the mass is large because the particle can only move via virtual fermionic states in which the pairing is broken $\llbracket$. As we shall show, this is not the case for an extended boson such as the $d$-wave pair discussed in this article, and the suppression of $T_{\mathrm{BE}}$ disappears.

Phenomenological models with resonance bosons have been proposed by Friedberg and Lee [5] and by Micnas, Ranninger, and Robaszkiewicz [6]. The $s$-wave description given in [5] can be extended to include the possibility of resonances having non-trivial symmetry. In the present paper, we discuss the formation of a two-fermion resonance ( $s$-wave or $d$-wave) and show that such a resonance boson can propagate long distances before decaying.

The present article is organized as follows: In Section III, we describe the fermionic Hamiltonian and present a theorem concerning the symmetry of the two-fermion ground state. Deep bound states, shallow bound states, and resonances are analyzed in Sections III, IV, and V. The discussion in these sections is mostly restricted to the case of a square lattice, with the exception of Section III A, in which we deal with a face-centered cubic lattice as in fullerene superconductors. Concluding remarks are made in Section V1. 


\section{TWO FERMIONS ON A PERIODIC LATTICE}

\section{A. The Hamiltonian and its properties}

We consider a periodic lattice of $\mathcal{N}$ sites with symmetry under inversion at each site. The fermionic kinetic energy is represented by a sum of "hopping" terms,

$$
H_{0}=\sum_{s, \mathbf{r}, \mathbf{r}^{\prime}} t\left(\mathbf{r}-\mathbf{r}^{\prime}\right) a_{s}^{\dagger}\left(\mathbf{r}^{\prime}\right) a_{s}(\mathbf{r})
$$

with $s=\uparrow$ (spin up) and $\downarrow$ (spin down). The interaction between two particles is described in terms of a potential energy that is a function of the relative separation and the total spin of a pair:

$$
\begin{aligned}
H_{1}=\sum_{\mathbf{r}, \mathbf{r}^{\prime}} & {\left[g_{S}\left(\mathbf{r}-\mathbf{r}^{\prime}\right) \mathcal{S}\left(\mathbf{r}, \mathbf{r}^{\prime}\right)^{\dagger} \mathcal{S}\left(\mathbf{r}, \mathbf{r}^{\prime}\right)\right.} \\
& \left.+g_{T}\left(\mathbf{r}-\mathbf{r}^{\prime}\right) \sum_{\mu} \mathcal{T}_{\mu}^{\dagger}\left(\mathbf{r}, \mathbf{r}^{\prime}\right) \mathcal{T}_{\mu}\left(\mathbf{r}, \mathbf{r}^{\prime}\right)\right],
\end{aligned}
$$

where $g_{S}(\mathbf{r})$ and $g_{T}(\mathbf{r})$ represent interaction energies in singlet and triplet states with a relative separation $\mathbf{r}$ and where $\mathcal{S}\left(\mathbf{r}, \mathbf{r}^{\prime}\right)$ and $\mathcal{T}_{\mu}\left(\mathbf{r}, \mathbf{r}^{\prime}\right)$ are the destruction operators for spin-singlet and spin-triplet states on sites $\mathbf{r}$ and $\mathbf{r}^{\prime}$, that is,

$$
\begin{aligned}
\mathcal{S}\left(\mathbf{r}, \mathbf{r}^{\prime}\right) & =\frac{a_{\uparrow}(\mathbf{r}) a_{\downarrow}\left(\mathbf{r}^{\prime}\right)+a_{\uparrow}\left(\mathbf{r}^{\prime}\right) a_{\downarrow}(\mathbf{r})}{2}, \\
\mathcal{T}_{0}\left(\mathbf{r}, \mathbf{r}^{\prime}\right) & =\frac{a_{\uparrow}(\mathbf{r}) a_{\downarrow}\left(\mathbf{r}^{\prime}\right)-a_{\uparrow}\left(\mathbf{r}^{\prime}\right) a_{\downarrow}(\mathbf{r})}{2}, \\
\mathcal{T}_{+}\left(\mathbf{r}, \mathbf{r}^{\prime}\right) & =\frac{a_{\uparrow}(\mathbf{r}) a_{\uparrow}\left(\mathbf{r}^{\prime}\right)}{\sqrt{2}}, \\
\mathcal{T}_{-}\left(\mathbf{r}, \mathbf{r}^{\prime}\right) & =\frac{a_{\downarrow}(\mathbf{r}) a_{\downarrow}\left(\mathbf{r}^{\prime}\right)}{\sqrt{2}} .
\end{aligned}
$$

The particular normalization of these operators has been chosen for later convenience. In this paper we are mainly concerned with singlet states. Therefore, the triplet part of the interaction will not contribute, and the subscript in the interaction energy $g_{S}$ will usually be suppressed.

It follows from (2.1) that the one-fermion energy spectrum is

$$
\epsilon_{\mathbf{p}}=\sum_{\mathbf{r}} t(\mathbf{r}) \cos (\mathbf{p} \cdot \mathbf{r})
$$

with $\mathbf{p}$ the Bloch momentum. The corresponding effective mass in $D$ dimensions is given by

$$
\frac{1}{m}=-\frac{1}{D} \sum_{\mathbf{r}} t(\mathbf{r}) r^{2}
$$

for the case in which the inverse mass-matrix $\left(m^{-1}\right)_{i j}=\delta_{i j} / m$. In this paper, we only consider the case for which $m>0$.

A convenient basis set in the Hilbert space of two fermions consists of states with total Bloch momentum $\mathbf{P}$, relative separation $\mathbf{r}$, and spin projections $s_{1}$ and $s_{2}$ :

$$
\left|\mathbf{P}, \mathbf{r}, s_{1}, s_{2}\right\rangle=\frac{1}{\sqrt{\mathcal{N}}} \sum_{\mathbf{r}^{\prime}} e^{i \mathbf{P} \cdot\left(\mathbf{r}^{\prime}+\mathbf{r} / 2\right)} a_{s_{1}}^{\dagger}\left(\mathbf{r}^{\prime}\right) a_{s_{2}}^{\dagger}\left(\mathbf{r}^{\prime}+\mathbf{r}\right)|0\rangle .
$$

Applying the kinetic energy operator (2.1) to state (2.6), we obtain

$$
H_{0}\left|\mathbf{P}, \mathbf{r}, s_{1}, s_{2}\right\rangle=\sum_{\mathbf{r}^{\prime}} 2 t\left(\mathbf{r}^{\prime}\right) \cos \left(\mathbf{P} \cdot \mathbf{r}^{\prime} / 2\right)\left|\mathbf{P}, \mathbf{r}+\mathbf{r}^{\prime}, s_{1}, s_{2}\right\rangle .
$$


The problem of two fermions is therefore reduced to that of a single particle hopping over $\mathbf{r}$ sites (in the relative position space) with an amplitude $2 t(\mathbf{r}) \cos (\mathbf{P} \cdot \mathbf{r} / 2)$. Eigenvectors of $H_{0}$ are states with total momentum $\mathbf{P}$ and definite relative momentum $\mathbf{p}$,

$$
\left|\mathbf{P}, \mathbf{p}, s_{1}, s_{2}\right\rangle=a_{(\mathbf{P} / 2)-\mathbf{p}, s_{1}}^{\dagger} a_{(\mathbf{P} / 2)+\mathbf{p}, s_{2}}^{\dagger}|0\rangle,
$$

with eigenvalues

$$
\begin{aligned}
E_{\mathbf{P}, \mathbf{p}} & =\epsilon_{(\mathbf{P} / 2)+\mathbf{p}}+\epsilon_{(\mathbf{P} / 2)-\mathbf{p}} \\
& =2 \sum_{\mathbf{r}} t(\mathbf{r}) \cos (\mathbf{P} \cdot \mathbf{r} / 2) \cos (\mathbf{p} \cdot \mathbf{r}) .
\end{aligned}
$$

The operators $a_{\mathbf{p}, s}$ and $a_{s}(\mathbf{r})$ are related by a Fourier transformation,

$$
a_{\mathbf{p}, s}=\frac{1}{\sqrt{\mathcal{N}}} \sum_{\mathbf{r}} e^{-i \mathbf{p} \cdot \mathbf{r}} a_{s}(\mathbf{r})
$$

The projection of any two-fermion state on the state (2.6) is the wave function in relative position space:

$$
\psi(\mathbf{r})=\left\langle\mathbf{P}, \mathbf{r}, s_{1}, s_{2} \mid \mathbf{P}, \psi, s_{1}, s_{2}\right\rangle .
$$

Plane waves,

$$
\frac{1}{\sqrt{\mathcal{N}}} \psi(\mathbf{r} \mid \mathbf{p}) \equiv\left\langle\mathbf{P}, \mathbf{r}, s_{1}, s_{2} \mid \mathbf{P}, \mathbf{p}, s_{1}, s_{2}\right\rangle=\frac{1}{\sqrt{\mathcal{N}}} e^{i \mathbf{p} \cdot \mathbf{r}},
$$

form a complete orthonormal set of functions:

$$
\begin{aligned}
& \frac{1}{\mathcal{N}} \sum_{\mathbf{p}} \psi^{*}\left(\mathbf{r}^{\prime} \mid \mathbf{p}\right) \psi(\mathbf{r} \mid \mathbf{p})=\delta_{\mathbf{r}^{\prime} \mathbf{r}}, \\
& \frac{1}{\mathcal{N}} \sum_{\mathbf{r}} \psi\left(\mathbf{r} \mid \mathbf{p}^{\prime}\right) \psi^{*}(\mathbf{r} \mid \mathbf{p})=\delta_{\mathbf{p}^{\prime} \mathbf{p}} .
\end{aligned}
$$

For singlet states, the two-fermion wave function must be symmetric under the transformation $\mathbf{r} \rightarrow-\mathbf{r}$ :

$$
\frac{1}{\sqrt{\mathcal{N}}} \psi(\mathbf{r} \mid \mathbf{p})=\langle\mathbf{P}, \mathbf{r} \mid \mathbf{P}, \mathbf{p}\rangle=\sqrt{\frac{2}{\mathcal{N}}} \cos (\mathbf{p} \cdot \mathbf{r})
$$

for $\mathbf{r} \neq 0$. For two fermions on the same site $(\mathbf{r}=0), \psi(0 \mid \mathbf{p})=1$ is already symmetric. Using the symmetrized wave functions (2.15), we can write the interaction matrix elements of $H_{1}$ as

$$
\left\langle\mathbf{P}, \mathbf{q}\left|H_{1}\right| \mathbf{P}, \mathbf{p}\right\rangle=\frac{1}{\mathcal{N}} \sum_{\mathbf{r}} \psi^{*}(\mathbf{r} \mid \mathbf{q}) g(\mathbf{r}) \psi(\mathbf{r} \mid \mathbf{p}) .
$$

Sites $\mathbf{r}$ and $-\mathbf{r}$ are now considered equivalent and only one of them enters the sum.

\section{B. Schrödinger equation}

To solve the Schrödinger equation,

$$
\left(H_{0}+H_{1}\right)|\mathbf{P}, \psi\rangle=E|\mathbf{P}, \psi\rangle,
$$

for a two-particle bound state, we write $|\mathbf{P}, \psi\rangle$ as a sum over states having definite relative momenta:

$$
|\mathbf{P}, \psi\rangle=\sum_{\mathbf{p}} F(\mathbf{p})|\mathbf{P}, \mathbf{p}\rangle .
$$

The Schrödinger equation is then transformed into an integral equation for $F(\mathbf{p})$ : 


$$
\begin{aligned}
\left(E-E_{\mathbf{P}, \mathbf{p}}\right) F(\mathbf{p}) & =\sum_{\mathbf{q}} F(\mathbf{q})\left\langle\mathbf{P}, \mathbf{p}\left|H_{1}\right| \mathbf{P}, \mathbf{q}\right\rangle \\
& =\frac{1}{\mathcal{N}} \sum_{\mathbf{r}} \psi^{*}(\mathbf{r} \mid \mathbf{p}) g(\mathbf{r}) \sum_{\mathbf{q}} F(\mathbf{q}) \psi(\mathbf{r} \mid \mathbf{q}) .
\end{aligned}
$$

Recalling that $\psi(\mathbf{r})=\sum_{\mathbf{q}} F(\mathbf{q}) \psi(\mathbf{r} \mid \mathbf{q})$, we obtain the following equation for the wave function:

$$
\psi(\mathbf{r})=\sum_{\mathbf{r}^{\prime}} G\left(E, \mathbf{P} ; \mathbf{r}, \mathbf{r}^{\prime}\right) g\left(\mathbf{r}^{\prime}\right) \psi\left(\mathbf{r}^{\prime}\right),
$$

where the Green's function $G$ is defined by

$$
G\left(E, \mathbf{P} ; \mathbf{r}, \mathbf{r}^{\prime}\right)=\frac{1}{\mathcal{N}} \sum_{\mathbf{q}} \frac{\psi(\mathbf{r} \mid \mathbf{q}) \psi^{*}\left(\mathbf{r}^{\prime} \mid \mathbf{q}\right)}{E-E_{\mathbf{P}, \mathbf{q}}} .
$$

In what follows, we shall truncate the interaction potential so that $g(\mathbf{r})$ vanishes beyond a certain distance, that is, $g(\mathbf{r}) \neq 0$ only when $\mathbf{r}=0, \pm \mathbf{R}_{1}, \ldots, \pm \mathbf{R}_{n-1}$. Equation $(2.20)$ for these sites can then be cast into matrix form:

$$
[1-\mathcal{G}(E, \mathbf{P}) g] \psi=0,
$$

where $\psi$ is an $n \times 1$ matrix with $\psi_{j}=\psi\left(\mathbf{R}_{j}\right), \mathcal{G}(E, \mathbf{P})$ is an $n \times n$ matrix with $\mathcal{G}_{i j}(E, \mathbf{P})=G\left(E, \mathbf{P} ; \mathbf{R}_{i}, \mathbf{R}_{j}\right)$, and $g$ is a diagonal $n \times n$ matrix with $g_{i i}=g\left(\mathbf{R}_{i}\right)$. Note that the kinetic energy of two particles (2.9) can also be written in matrix form,

$$
\begin{aligned}
E_{\mathbf{P}, \mathbf{p}} & =\epsilon_{(\mathbf{P} / 2)+\mathbf{p}}+\epsilon_{(\mathbf{P} / 2)-\mathbf{p}} \\
& =2 \sum_{i, j} \psi_{i}^{*}(\mathbf{P} / 2) \tau_{i j} \psi_{j}(\mathbf{p}),
\end{aligned}
$$

where the diagonal matrix $\tau$ is defined by $\tau_{i i}=t\left(\mathbf{R}_{i}\right)$ and where $\psi_{i}(\mathbf{p}) \equiv \psi\left(\mathbf{R}_{i} \mid \mathbf{p}\right)$. It follows from Equation (2.22) that the eigenvalues $E$ are determined by the secular equation,

$$
\operatorname{det}\left[\mathcal{G}(E, \mathbf{P})-g^{-1}\right]=0 .
$$

For each eigenvalue $E$, the wave function at the $n$ sites $\psi\left(\mathbf{R}_{j}\right)$ is determined by Equation (2.22), while for sites beyond the interaction range one uses Equation (2.20) to determine $\psi(\mathbf{r})$.

In the case of a scattering process, the solution to the Schrödinger equation (2.17) with an incident plane wave $\psi(\mathbf{r} \mid \mathbf{p})$ satisfies

$$
\psi(\mathbf{r})=\psi(\mathbf{r} \mid \mathbf{p})+\sum_{\mathbf{r}^{\prime}} G\left(E, \mathbf{P} ; \mathbf{r}, \mathbf{r}^{\prime}\right) g\left(\mathbf{r}^{\prime}\right) \psi\left(\mathbf{r}^{\prime}\right)
$$

$\psi(\mathbf{r})$ is therefore given by

$$
\begin{aligned}
\psi(\mathbf{r}) & =\psi(\mathbf{r} \mid \mathbf{p}) \\
& +\sum_{i=0}^{n} \sum_{j=0}^{n} G\left(E, \mathbf{P} ; \mathbf{r}, \mathbf{R}_{i}\right) \mathcal{A}_{i j}(E, \mathbf{P}) \psi\left(\mathbf{R}_{j} \mid \mathbf{p}\right),
\end{aligned}
$$

where the $n \times n$ scattering matrix $\mathcal{A}(E, \mathbf{P})$ is defined by the equation]

$$
\mathcal{A}^{-1}(E, \mathbf{P})=g^{-1}-\mathcal{G}(E, \mathbf{P}) .
$$

$\mathcal{A}(E, \mathbf{P})$, as a function of complex $E$, has a cut along the real axis corresponding to the continuum spectrum of two free fermions. Depending on the potential $g(\mathbf{r})$, it may also have poles on the real axis (bound states) or below the upper edge of the cut (resonances).

\footnotetext{
${ }^{1}$ It should be noted that $\mathcal{A}_{i j} \neq\left. A\left(\mathbf{r}, \mathbf{r}^{\prime}\right)\right|_{\mathbf{r}=\mathbf{R}_{i}, \mathbf{r}^{\prime}=\mathbf{R}_{j}}$, where $A$ is the scattering matrix defined on the entire lattice. It is true, however, that $\mathcal{A}^{-1}{ }_{i j}=\left.A^{-1}\left(\mathbf{r}, \mathbf{r}^{\prime}\right)\right|_{\mathbf{r}=\mathbf{R}_{i}, \mathbf{r}^{\prime}=\mathbf{R}_{j}}$.
} 


\section{Symmetry of the ground state}

We shall now demonstrate that the wave function of the ground state of two fermions does not change sign unless some of the hopping amplitudes $t(\mathbf{r})$ are positive. The situation is similar to that in the continuum, for which the ground-state wave function has no nodes. Note that a positive effective mass [Equation (2.5)] requires at least some of the hopping amplitudes to be negative.

We have seen that the problem of two fermions reduces to a one-body problem with modified hopping amplitudes. It therefore suffices to study the ground state of a single particle moving on the same lattice in the external potential $g(\mathbf{r})$. We consider the case of total momentum $\mathbf{P}=0$, for which the amplitudes double (reminiscent of the reduced mass effect in the continuum). The ground state is obtained by minimizing the expectation value of the energy:

$$
\langle E\rangle=\sum_{\mathbf{r}, \mathbf{r}^{\prime}} 2 t\left(\mathbf{r}-\mathbf{r}^{\prime}\right) \psi^{*}\left(\mathbf{r}^{\prime}\right) \psi(\mathbf{r})+\sum_{\mathbf{r}} g(\mathbf{r})|\psi(\mathbf{r})|^{2} .
$$

Suppose that $t(\mathbf{r}) \leq 0$ for all $\mathbf{r}$ and that $\psi(\mathbf{r})$ is positive on some site $\mathbf{r}_{1}$ and negative on another site $\mathbf{r}_{2}$. Then the first term in (2.28) can be lowered if instead of a negative $\psi\left(\mathbf{r}_{2}\right)$ we use $\left|\psi\left(\mathbf{r}_{2}\right)\right|$, the second term remaining unaffected by such a change. Therefore, the absolute minimum of $\langle E\rangle$ must have $\psi(\mathbf{r}) \geq 0$ on all sites, provided there always exists a sequence of allowed hops from any one site to any other site on the lattice.

\section{DEEP BOUND STATES}

When the magnitude of an attractive potential exceeds the kinetic energy of two fermions, compact pairs are formed and can be treated as small-size bosons. Pairs formed by two fermions on the same site, however, have a vanishing mobility in the limit of strong binding. This strongly decreases the condensation temperature 《], in contrast to the continuum case. It is desirable to have a model which does not have this lattice artifact. The pairing of fermions on adjacent sites is one way around the difficulty. We address this issue by working out two specific examples of lattice fermions having a strong nearest-neighbor attraction: first on a face-centered cubic lattice and then on a square lattice.

The advantage of extended-boson models is offset by a new problem, which is also a lattice effect. While only two fermions can be involved in on-site attractive interactions due to the Pauli principle, extended-range attractive potentials can result in the formation of many-body bound states. However, quantum statistics can help avoid this type of phase separation because the interaction strength in symmetric (spin-singlet) and antisymmetric (spin-triplet) spatial states can be quite different. Let us assume that the interaction is attractive in a singlet state and repulsive in a triplet. Because a fermion cannot form more than one singlet bond at a time, it will attract only one other fermion. More exactly, quantum spin fluctuations will produce a fluctuating potential between fermions. Only in pairs are these fluctuations completely suppressed, and a state with pairs may be favored over other many-body states. For details, see Appendix A.

\section{A. Deep bound states on a face-centered cubic lattice}

The following simple model describes tightly bound fermion pairs with a small effective mass (close to that of individual fermions). The small mass results from fermionic pairs which move through the lattice without having to break the bond. Triangular and f.c.c. lattices offer such a possibility.

The Hamiltonian [Equations (2.1) and (2.2)] for the f.c.c. model contains nearest-neighbor hopping with amplitude $t$, on-site repulsion $g_{0}$, and attraction $g_{1}$ for fermions on adjacent sites. On an f.c.c. lattice, there are twelve nearest neighbors $\mathbf{r}^{\prime}$ around any one site $\mathbf{r}$, with

$$
\mathbf{r}^{\prime}-\mathbf{r}=\frac{a}{2}( \pm \hat{\mathbf{x}} \pm \hat{\mathbf{y}}), \frac{a}{2}( \pm \hat{\mathbf{y}} \pm \hat{\mathbf{z}}), \frac{a}{2}( \pm \hat{\mathbf{z}} \pm \hat{\mathbf{x}})
$$

$\hat{\mathbf{x}}, \hat{\mathbf{y}}$, and $\hat{\mathbf{z}}$ are the unit vectors along each side of the cube and $a$ is the length of each side.

If $\left|g_{1}\right| \gg|t|$, so that the bond between fermions is strong, the relative-position wave function $\psi(\mathbf{r}) \neq 0$ only on the 12 sites surrounding the origin. We therefore expect 12 bound states with the same potential energy $g_{1}$ and, in general, unequal kinetic energies at a total Bloch momentum $\mathbf{P} \neq 0$. The kinetic energy is determined by the hopping amplitudes, $2 t \cos (\mathbf{P} \cdot \mathbf{r} / 2)$, as discussed in Section II A. 
At $\mathbf{P}=0$, all hopping amplitudes are $2 t$ and the energy levels have degeneracy (see Appendix B): there are a singlet $A_{1}$, a doublet $E$, and a triplet $T_{2}$ (Figure 1 ). As long as $t<0$, the ground state for $\mathbf{P}=0$ is $A_{1}$, with $\psi(\mathbf{r})=1 / \sqrt{12}$ on the 12 sites around the origin and zero otherwise. From each of these 12 sites a fermion can hop to 4 of its nearest neighbors (see Figure $1(\mathrm{a})$ ). For $\mathbf{P} \neq 0$, the boson dispersion relation to lowest order in $\mathbf{P}$ is given by expanding $\cos (\mathbf{P} \cdot \mathbf{r} / 2)$ to second order:

$$
E(\mathbf{P})=g_{1}+8 t-\frac{t}{12} \sum_{\mathbf{r}, \mathbf{r}^{\prime \prime}}^{\mathrm{n} . \mathrm{n} .}\left[\frac{\mathbf{P} \cdot\left(\mathbf{r}-\mathbf{r}^{\prime \prime}\right)}{2}\right]^{2},
$$

where $\mathbf{r}^{\prime \prime}$ denotes the four nearest-neighbor sites shown in Figure 1(a). Because $E$ is an isotropic function of $\mathbf{P}$ to this order, we average over all directions of the total momentum and substitute $\left|\mathbf{r}-\mathbf{r}^{\prime \prime}\right|=a / \sqrt{2}$ to obtain

$$
E(\mathbf{P})=g_{1}+8 t-\frac{t P^{2} a^{2}}{6}
$$

which gives the effective mass of a boson as

$$
m_{b}=-\frac{3 \hbar^{2}}{t a^{2}}=6 m_{f}
$$

\section{B. Deep bound states on a square lattice.}

On a square lattice, the mass of a strongly bound pair will be finite if one takes into account next-nearest-neighbor fermion hopping (with amplitude $t_{2}$ ) in addition to nearest-neighbor hopping (with amplitude $t_{1}$ ). Suppose the interaction potential has strength $g_{0}>0$ for particles on the same site, $g_{1}<0$ for nearest neighbors, $g_{2}>0$ for nextnearest neighbors, and 0 otherwise. For spin singlets, we have five distinct relative positions with non-zero interactions: $\mathbf{R}_{0}=(0,0), \mathbf{R}_{1}=(0, \pm a), \mathbf{R}_{2}=( \pm a, 0), \mathbf{R}_{3}=( \pm a, \pm a)$, and $\mathbf{R}_{4}=( \pm a, \mp a)$. In this basis, $\tau=\operatorname{diag}\left(0, t_{1}, t_{1}, t_{2}, t_{2}\right)$ and $V=\operatorname{diag}\left(g_{0}, g_{1}, g_{1}, g_{2}, g_{2}\right)$. According to Equation (2.5), the fermion mass is

$$
m_{f}=\frac{\hbar^{2}}{2\left(-t_{1}-2 t_{2}\right) a^{2}}
$$

As in the determination of a single fermion mass, we expand the energy of a two-fermion bound state in powers of $\mathbf{P}$. Near $\mathbf{P}=0$, it is convenient to use the irreducible representations of the symmetry group (see Appendix B); and we choose the following five components $\psi_{i}(\mathbf{p})$,

$$
\psi(\mathbf{p}) \equiv\left(\begin{array}{c}
\psi_{0}(\mathbf{p}) \\
\psi_{1}(\mathbf{p}) \\
\psi_{2}(\mathbf{p}) \\
\psi_{3}(\mathbf{p}) \\
\psi_{4}(\mathbf{p})
\end{array}\right)=\left(\begin{array}{c}
1 \\
\cos p_{x} a+\cos p_{y} a \\
\cos p_{x} a-\cos p_{y} a \\
2 \cos p_{x} a \cos p_{y} a \\
2 \sin p_{x} a \sin p_{y} a
\end{array}\right)
$$

as a basis set. Functions $\psi_{0}, \psi_{1}$, and $\psi_{3}$ belong to the $A_{1}$ representation (refered to as $s$-wave in the literature); $\psi_{2}$ transforms as $B_{1}\left(d_{x^{2}-y^{2}}\right.$-wave); and $\psi_{4}$ as $B_{2}$ ( $d_{x y}$-wave). Assuming that the nearest-neighbor attraction $g_{1}$ is strong and $E \approx g_{1}$, we expand the Green's function

$$
G_{i j}(E, \mathbf{P}) \equiv \frac{1}{\mathcal{N}} \sum_{\mathbf{p}} \frac{\psi_{i}(\mathbf{p}) \psi_{j}^{*}(\mathbf{p})}{E-2 \sum_{k, l=0}^{4} \psi_{k}^{*}(\mathbf{P} / 2) \tau_{k l} \psi_{l}(\mathbf{p})}
$$

in powers of $1 / E$ and thus determine the dependence $E(\mathbf{P})$. Because

$$
G_{i j}(E, \mathbf{P})=\frac{\delta_{i j}}{E}+\mathcal{O}\left(\frac{1}{E^{2}}\right),
$$

we need only the diagonal matrix elements to calculate the lowest-order correction to $E$. We have 


$$
\begin{gathered}
G_{00}(E, \mathbf{P})=G_{33}(E, \mathbf{P})=G_{44}(E, \mathbf{P})=\frac{1}{E}+\mathcal{O}\left(\frac{1}{E^{3}}\right), \\
G_{11}(E, \mathbf{P})=\frac{1}{E}+\frac{2 t_{2}}{E^{2}} \psi_{3}(\mathbf{P} / 2)+\mathcal{O}\left(\frac{1}{E^{3}}\right), \\
G_{22}(E, \mathbf{P})=\frac{1}{E}-\frac{2 t_{2}}{E^{2}} \psi_{3}(\mathbf{P} / 2)+\mathcal{O}\left(\frac{1}{E^{3}}\right) .
\end{gathered}
$$

Upon substituting these expressions into equation (2.24), we obtain the following dispersion laws:

$$
E=g_{1} \pm 2 t_{2} \psi_{3}(\mathbf{P} / 2)+\mathcal{O}\left(\frac{1}{g_{1}}\right),
$$

where the plus sign corresponds to the $A_{1}$ state $\psi_{1}$ and the minus sign corresponds to the $B_{1}$ state $\psi_{2}$. The other three bands have no dispersion to this order. For a positive (negative) $t_{2}$, the ground state transforms according to representation $B_{1}\left(A_{1}\right)$ and the mass of the boson is determined strictly by the diagonal hopping term:

$$
m_{b}=\frac{\hbar^{2}}{\left|t_{2}\right| a^{2}}=\frac{-2 t_{1}-4 t_{2}}{\left|t_{2}\right|} m_{f}
$$

Note that, unlike in the case of a strong on-site attraction, an extended boson can easily move through the lattice without breaking its bond, hence a small mass even for a strong attraction. It can even be smaller than the mass of its constituent fermions.

It is straightforward to see what determines the symmetry of the ground state. The relative position of two fermions is restricted to four nearest-neighbor sites by a strong attraction. Depending on the sign of $t_{2}$, the wavefunction must either alternate its sign on these sites or maintain its sign to minimize the kinetic energy.

\section{SHALLOW BOUND STATES}

In this section, we investigate the properties of bound states that lie close to the bottom of the two-fermion band, which is assumed to have a single minimum $E_{0}$ at the relative momentum $\mathbf{p}=0$. As described in Section II B, we first solve Equation (2.22) on the relative sites having a non-zero interaction potential. As before, we assume that $g(\mathbf{r}) \neq 0$ only for a finite number of lattice sites $\mathbf{r}=0, \pm \mathbf{R}_{1}, \ldots, \pm \mathbf{R}_{n-1}$. Let us also assume that the same-site repulsion is infinite so that $\psi(0)=0$. Using Greek indices to enumerate sites $\pm \mathbf{R}_{1}, \ldots, \pm \mathbf{R}_{n-1}$, we obtain from Equation (2.22) the following set of equations for the vector $\Delta=g \psi$ :2

$$
\begin{aligned}
\mathcal{G}_{00} \Delta_{0}+\mathcal{G}_{0 \alpha} \Delta_{\alpha} & =g^{-1}{ }_{00} \Delta_{0}=0, \\
\mathcal{G}_{\alpha 0} \Delta_{0}+\mathcal{G}_{\alpha \beta} \Delta_{\beta} & =g^{-1}{ }_{\alpha \beta} \Delta_{\beta} .
\end{aligned}
$$

Summation over repeated indices is assumed. Eliminating $\Delta_{0}$, we obtain

$$
\left(\mathcal{G}_{\alpha \beta}-\frac{\mathcal{G}_{\alpha 0} \mathcal{G}_{0 \beta}}{\mathcal{G}_{00}}\right) \Delta_{\beta}=g^{-1}{ }_{\alpha \beta} \Delta_{\beta}
$$

Now we can use Equation (2.20) to calculate the wave function on the remainder of the lattice:

$$
\begin{aligned}
\psi(\mathbf{r}) & =G\left(\mathbf{r}, \mathbf{R}_{i}\right) \Delta_{i} \\
& =\left[G\left(\mathbf{r}, \mathbf{R}_{\alpha}\right)-\frac{G(\mathbf{r}, 0) G\left(0, \mathbf{R}_{\alpha}\right)}{G(0,0)}\right] \Delta_{\alpha} .
\end{aligned}
$$

\footnotetext{
${ }^{2}$ The BCS gap $\Delta(\mathbf{r})$ is the second-quantized counterpart of this function.
} 
Apart from a multiplicative constant, the long-range behavior of $\psi(\mathbf{r})$ is determined by the properties of the Green's function at large separations, which in turn is controlled by the long-wavelength behavior of the summand in Equation (2.21). The latter diverges at $\mathbf{q}=0$ as energy $E$ approaches the bottom of the two-fermion band. Then the denominator in (2.21) can be approximated using the effective fermion mass:

$$
E-E_{\mathbf{P}, \mathbf{q}} \approx E-E_{\mathbf{P}, 0}-\frac{q^{2}}{m_{f}}=-\frac{q^{2}+1 / \xi_{0}^{2}}{m_{f}},
$$

which defines a coherence length $\xi_{0}$ at themperature $T=0$ as the size of the bound state. For $r \gg \xi_{0}$, the propagator decays exponentially with the distance, while in the intermediate range, $a \ll r \ll \xi_{0}$, it decreases as a power of $\left|\mathbf{r}-\mathbf{r}^{\prime}\right|$.

It is possible to isolate the divergent part of the Green's function in the form of $G(E, \mathbf{P} ; 0,0) \equiv G(0,0)$. We define a regularized Green's function as follows. For $\mathbf{r} \neq 0$ and $\mathbf{r}^{\prime} \neq 0$,

$$
\begin{gathered}
F\left(\mathbf{r}, \mathbf{r}^{\prime}\right)=\frac{1}{\mathcal{N}} \sum_{\mathbf{q}} \frac{[\psi(\mathbf{r} \mid \mathbf{q})-\psi(\mathbf{r} \mid 0)]\left[\psi^{*}\left(\mathbf{r}^{\prime} \mid \mathbf{q}\right)-\psi^{*}\left(\mathbf{r}^{\prime} \mid 0\right)\right]}{E-E_{\mathbf{P}, \mathbf{q}}} \\
F(\mathbf{r}, 0)=\frac{1}{\mathcal{N}} \sum_{\mathbf{q}} \frac{[\psi(\mathbf{r} \mid \mathbf{q})-\psi(\mathbf{r} \mid 0)] \psi^{*}(0 \mid \mathbf{q})}{E-E_{\mathbf{P}, \mathbf{q}}} \\
F\left(0, \mathbf{r}^{\prime}\right)=\frac{1}{\mathcal{N}} \sum_{\mathbf{q}} \frac{\psi(0 \mid \mathbf{q})\left[\psi\left(\mathbf{r}^{\prime} \mid \mathbf{q}\right)-\psi^{*}\left(\mathbf{r}^{\prime} \mid 0\right)\right]}{E-E_{\mathbf{P}, \mathbf{q}}}
\end{gathered}
$$

These sums are regular at $\mathbf{q}=0$. By inspection,

$$
\begin{aligned}
G(\mathbf{r}, 0)= & \psi(\mathbf{r} \mid 0) G(0,0)+F(\mathbf{r}, 0) \\
G\left(0, \mathbf{r}^{\prime}\right)= & G(0,0) \psi^{*}\left(\mathbf{r}^{\prime} \mid 0\right)+F\left(0, \mathbf{r}^{\prime}\right) \\
G\left(\mathbf{r}, \mathbf{r}^{\prime}\right)= & \psi(\mathbf{r} \mid 0) G(0,0) \psi^{*}\left(\mathbf{r}^{\prime} \mid 0\right)+\psi(\mathbf{r} \mid 0) F\left(0, \mathbf{r}^{\prime}\right) \\
& +F(\mathbf{r}, 0) \psi^{*}\left(\mathbf{r}^{\prime} \mid 0\right)+F\left(\mathbf{r}, \mathbf{r}^{\prime}\right) .
\end{aligned}
$$

Upon substituting these functions into Equation (4.3), we finally obtain the wave function as a sum of two terms,

$$
\begin{aligned}
\psi(\mathbf{r})= & F\left(E, \mathbf{P} ; \mathbf{r}, \mathbf{R}_{\alpha}\right) \Delta_{\alpha} \\
& -F\left(E, \mathbf{P} ; 0, \mathbf{R}_{\alpha}\right) \Delta_{\alpha} \frac{G(E, \mathbf{P} ; \mathbf{r}, 0)}{G(E, \mathbf{P} ; 0,0)} .
\end{aligned}
$$

Consider states with total Bloch momentum $\mathbf{P}=0$. The first term in Equation (4.7) is proportional to the regularized propagator $F\left(E, \mathbf{P} ; \mathbf{r}, \mathbf{R}_{\alpha}\right)$, whose Fourier transform remains finite as $\mathbf{q} \rightarrow 0$. In the intermediate range, $a \ll r \ll \xi_{0}$, it decays with $\mathbf{r}$ as $r^{-D}$ or faster ( $D$ is the number of spatial dimensions). The second term, on the other hand, is on the order of $r^{2-D}$, as can be inferred from its singular Fourier transform. It therefore should dominate at large distances. However, this term vanishes if the state belongs to any irreducible representation other than the trivial one due to the prefactor $F\left(E, 0 ; 0, \mathbf{R}_{\alpha}\right) \Delta_{\alpha}$. $^{3}$ This means that a bound state which transforms according to a non-trivial irreducible representation of the point group will be smaller in size than a symmetric bound state of the same energy. This may have verifiable consequences for superconductivity.

To illustrate the importance of this point, we use a continuum approximation to the two-particle problem. In the intermediate region, $a \ll r \ll \xi_{0}$, the two-particle wavefunction $\psi(\mathbf{r})$ satisfies the continuum Laplace equation, $\nabla^{2} \psi(\mathbf{r})=0$. In 2 and 3 dimensions, solutions with angular momentum $l$ and its projection $m$ are

$$
\begin{aligned}
& \psi(\mathbf{r})=e^{i m \phi} r^{-m}(2 \text { dimensions }), \\
& \psi(\mathbf{r})=Y_{l m}(\theta, \phi) r^{-l-1}(3 \text { dimensions }) .
\end{aligned}
$$

\footnotetext{
${ }^{3}$ The Green's function $F\left(E, 0 ; 0, \mathbf{R}_{\alpha}\right)$ is invariant under all point group transformations.
} 
It is generally thought that the lattice spacing $a$ is irrelevant as long as we deal with a weakly bound pair $\left(\xi_{0} \gg a\right)$. A simple scaling argument demonstrates that this is not always the case. To imitate the infrared and ultraviolet cutoffs, we set $\psi(\mathbf{r})=0$ outside the interval $a<r<\xi_{0}$. In 3 dimensions, the $n$-th moment of $r$ is then given by

$$
\left\langle r^{n}\right\rangle=\frac{\int_{a}^{\xi_{0}} r^{-2 l-2+n} r^{2} d r}{\int_{a}^{\xi_{0}} r^{-2 l-2} r^{2} d r} .
$$

For $l=0$ one obtains

$$
\left\langle r^{n}\right\rangle=\frac{\xi_{0}^{n}}{n+1}
$$

for any $n \geq 0$. Indeed, an $s$ state is not sensitive to the ultraviolet cutoff (the lattice spacing). For $l \geq 1$,

$$
\begin{aligned}
& n<2 l-1: \quad\left\langle r^{n}\right\rangle=\frac{2 l-1}{2 l-1-n} a^{n}, \\
& n=2 l-1: \quad\left\langle r^{n}\right\rangle=(2 l-1) a^{2 l-1} \log \frac{\xi_{0}}{a}, \\
& n>2 l-1: \quad\left\langle r^{n}\right\rangle=\frac{2 l-1}{n-2 l+1} \xi_{0}^{n}\left(\frac{a}{\xi_{0}}\right)^{2 l-1} .
\end{aligned}
$$

This suggests that, for a shallow bound state with non-zero angular momentum, the measured size of a pair is determined by both length scales, $\xi_{0}$ and $a$, and therefore may be much smaller than $\xi_{0}$. The physical reason behind this difference is that, at the same value of kinetic energy, states with a larger angular momentum have a smaller radial momentum. Therefore, the ability to tunnel into the classically forbidden region (to distances on the order of $\left.\xi_{0}\right)$ is substantially reduced. Similar arguments hold for the two-dimensional case.

The small size of bosonic pairs may imply a high upper critical magnetic field. As has been argued in [7], the upper critical magnetic field for the condensate of an ideal charged Bose gas at zero temperature is infinite, because of the very different roles played by the Coulomb interaction in Bose and Fermi systems. Therefore, a rapid increase of the critical field as $T \rightarrow 0$ should be expected for an ideal charged Bose gas. Recently, a similar behavior has been observed in $T_{c}$-suppressed cuprates [8]. This observation indicates that Bose condensation may be dominant in these superconductors. Of course, bosons in real systems are not point particles. A rough estimate of the upper critical field would be the magnetic field at which the cyclotron radius of fermions is comparable to the size of d-wave pairs,

$$
\phi_{0}=2 \pi H_{\mathrm{c} 2}\left\langle r^{2}\right\rangle
$$

where $\phi_{0}=h c / 2 e$ is a flux quantum. Formally, this criterion reproduces the Landau-Ginzburg result, provided that the pair size is replaced by the coherence length $\xi$. We stress, however, that in a $d$-wave superconductor the former can be smaller than the latter.

We now investigate the model described in Section III B in order to determine the symmetry of a shallow ground state. Consider the case in which the bound state first appears below the continuum band. Using the basis set (3.6), we write the secular equation in the form of Equation (4.2). It is assumed that the on-site repulsion $g_{0}$ is infinite. For a state with an energy just at the bottom of the two-fermion band, it is convenient to use a regularized Green's function $\mathcal{F}(E, \mathbf{P})$ as was introduced previously. Equation 4.2 ) then reads

$$
\left(\mathcal{F}_{\alpha \beta}-\frac{\mathcal{F}_{\alpha 0} \mathcal{F}_{0 \beta}}{\mathcal{G}_{00}}\right) \Delta_{\beta}=g^{-1}{ }_{\alpha \beta} \Delta_{\beta} .
$$

$\mathcal{G}_{00}$ becomes infinite as $E \rightarrow 2 \epsilon_{0}$, which further simplifies the problem. The critical values of the coupling strength are then given by the equation

$$
\left(\mathcal{F}_{\alpha \beta}-g_{\alpha \beta}^{-1}\right) \Delta_{\beta}=0
$$

where $\mathcal{F}_{\alpha \beta}$ is to be evaluated at $\mathbf{P}=0$ and $E=2 \epsilon_{0}$. For the irreducible representations $A_{1}, B_{1}$, and $B_{2}$; one obtains, respectively,

$$
\begin{aligned}
\left(\mathcal{F}_{11}-g_{1}^{-1}\right)\left(\mathcal{F}_{33}-g_{2}^{-1}\right) & =\mathcal{F}_{13} \mathcal{F}_{31}, \\
\mathcal{F}_{22}-g_{1}^{-1} & =0 \\
\mathcal{F}_{44}-g_{2}^{-1} & =0 .
\end{aligned}
$$


As long as $g_{2}$ represents repulsion, the $B_{2}$ bound state $\left(d_{x y}\right)$ does not exist.

If there is no next-nearest-neighbor repulsion $\left(g_{2}=0\right)$, it can be seen that the ground state is $A_{1}$ ( $s$ wave). Indeed, Equation (4.15a) reduces to $\mathcal{F}_{11}=g_{1}^{-1}$ and one obtains the following relation between the critical values of $g_{1}$ for $s$ and $d_{x^{2}-y^{2}}$ waves:

$$
\frac{1}{g_{1}^{(s)}}-\frac{1}{g_{1}^{(d)}}=\frac{2}{\mathcal{N}} \sum_{\mathbf{q}} \frac{\left(1-\cos q_{x} a\right)\left(1-\cos q_{y} a\right)}{\epsilon_{0}-\epsilon_{\mathbf{q}}}<0 .
$$

Thus, it takes a weaker attraction to produce an $s$-wave ground state when there is no next-nearest-neighbor repulsion.

When the next-nearest-neighbor amplitude $t_{2}$ is negative, the ground state is an $s$ wave, in accordance with the result of Section II . Therefore, the search for a non-trivial symmetry of the ground state is restricted to the domain in which both $t_{2}>0$ and $g_{2}>0$. The results can be summarized as follows. An increasing next-nearest-neighbor repulsion $g_{2}$ raises the kinetic energy of an $s$ wave but does not affect that of a $d_{x^{2}-y^{2}}$ wave. A positive $t_{2}$ means positive kinetic energy for $s$ waves and negative kinetic energy for $d$ waves. The actual "phase diagram" is presented in Figure 2. For the value of $t_{2}=0.45\left|t_{1}\right|$ inferred by Pines from fitting NMR results [3], we find that the strength of the next-nearest-neighbor repulsion must be at least $0.35\left|t_{1}\right|$ in order for the ground state to have the $\left(x^{2}-y^{2}\right)$ symmetry.

\section{EXTENDED RESONANCE STATES}

\section{A. General approach}

Even if the two-particle potential energy is positive at all separations, there still remains the possibility of two fermions forming a resonance state - a state bound for a limited time - provided that the potential has a local minimum. It has been shown [5] that resonance bosons can form a condensate, thus inducing superconductivity in the boson-fermion system.

On a lattice, we may approach the problem of a single resonance in the following way. When a local minimum of the potential is surrounded by a closed wall of sites with infinite repulsion, the wave function cannot leak out and two fermions form a stable bound state. Then the Schrödinger equation, $\left(H_{0}+H_{1}\right)|\mathbf{P}, \psi\rangle=E|\mathbf{P}, \psi\rangle$, has a solution for a real $E$ inside the two-fermion energy band. The wave function $\psi(\mathbf{r})$ is non-zero only for separations $\mathbf{r}$ within the wall, which makes the Schrödinger equation readily solvable. Its solution also satisfies Equation (2.20), which determines the location of the pole of the scattering amplitude. In the limit of a stable state, the residue of the pole vanishes, reflecting the fact that incident free particles cannot penetrate the barrier of infinite repulsion. Lowering the height of the wall to a finite value makes the localized two-fermion state unstable, thereby shifting the pole away from the real axis into the unphysical sheet. In what follows, we determine the mass and resonance width of a bosonic state to lowest orders in the inverse height of the wall.

Let the wave function of the stable state be $\psi(\mathbf{r})$, normalized to 1 . Outside the wall, $\psi(\mathbf{r})=0 . \psi(\mathbf{r})$ satisfies Equation $(2.20)$, which now must be modified due to the existence of a branch cut of the function $G\left(E, \mathbf{P} ; \mathbf{r}^{\prime}, \mathbf{r}\right)$ on the real axis of $E$ :

$$
\psi(\mathbf{r})=\sum_{\mathbf{r}^{\prime}} G\left(E \pm i 0^{+}, \mathbf{P} ; \mathbf{r}, \mathbf{r}^{\prime}\right) g\left(\mathbf{r}^{\prime}\right) \psi\left(\mathbf{r}^{\prime}\right)
$$

Because $\psi(\mathbf{r})$ does not contain incoming or outgoing free particles, it is invariant under time reversal and therefore satisfies this equation on both edges of the cut. The complex conjugate of (5.1a) is

$$
\psi^{*}(\mathbf{r})=\sum_{\mathbf{r}^{\prime}} \psi^{*}\left(\mathbf{r}^{\prime}\right) g\left(\mathbf{r}^{\prime}\right) G\left(E \mp i 0^{+}, \mathbf{P} ; \mathbf{r}^{\prime}, \mathbf{r}\right) .
$$

As before, let us assume that the inter-particle potential $g(\mathbf{r}) \neq 0$ only at the $2 n-1$ sites $\mathbf{r}=0, \pm \mathbf{R}_{1}, \ldots, \pm \mathbf{R}_{n-1}$. We introduce a new wave function $\Delta(\mathbf{r}) \equiv g(\mathbf{r}) \psi(\mathbf{r})$, which remains finite at a site $\mathbf{r}$ even as $g(\mathbf{r}) \rightarrow \infty$ as long as the state has finite energy. Using the matrix notations of Section IIB, we rewrite Equations 5.1a and 5.1b in terms of the $n \times 1$ column vector $\Delta$ :

$$
\begin{aligned}
{\left[g^{-1}-\mathcal{G}\left(E \pm i 0^{+}, \mathbf{P}\right)\right] \Delta } & =0 \\
\Delta^{\dagger}\left[g^{-1}-\mathcal{G}\left(E \mp i 0^{+}, \mathbf{P}\right)\right] & =0 .
\end{aligned}
$$


In what follows, we will omit the variables $E$ and $\mathbf{P}$ unless their presence is necessary to avoid ambiguity.

By regarding the diagonal matrix $g^{-1}$ as an independent variable, we can treat the position of the pole $E$ as a function of $g^{-1}$. When $g^{-1}(\mathbf{r})=0$ on the walls, $E$ is readily determined from the Schrödinger equation for the interior of the wall. By changing $g^{-1}$ infinitesimally, we can trace the trajectory of $E$ in the complex plane. Differentiate Equation (5.2a):

$$
\left(g^{-1}-\mathcal{G}\right) d \Delta+\left(d g^{-1}-\frac{\partial \mathcal{G}}{\partial E} d E\right) \Delta=0
$$

Multiplication of Equation (5.3) by $\Delta^{\dagger}$ eliminates the first term because of Equation (5.2b). Then,

$$
\Delta^{\dagger} \frac{\partial \mathcal{G}}{\partial E} \Delta d E=\Delta^{\dagger} d g^{-1} \Delta .
$$

The factor in front of $d E$ is merely -1 (see Appendix D).

We thus have the following expression for the first-order displacement of the pole:

$$
d E=-\Delta^{\dagger} d g^{-1} \Delta
$$

which is real. Formally, $d E$ can be written as $\psi^{\dagger} d g \psi$, the first-order term in the standard perturbation series, although the latter is not well defined for an infinite repulsion.

Next, from Equation (5.3) we obtain the first-order correction to $\Delta$ :

$$
\begin{aligned}
d \Delta & =-\mathcal{A}\left(d g^{-1}-\frac{\partial \mathcal{G}}{\partial E} d E\right) \Delta \\
& =-\mathcal{A}\left(d g^{-1} g+\mathcal{G} d E\right) \psi,
\end{aligned}
$$

where $\mathcal{A}^{-1}=g^{-1}-\mathcal{G}^{-1}$. Here, again, the substitution of $\partial \mathcal{G} / \partial E$ by $-\mathcal{G}^{2}$ requires justification (Appendix D).

To obtain the second-order correction to the energy of the pole $E$, we differentiate Equation (5.3) once again and multiply by $\Delta^{\dagger} / 2$ to eliminate the term with $d^{2} \Delta$ :

$$
\begin{aligned}
& -\frac{1}{2} \Delta^{\dagger}\left(\frac{\partial^{2} \mathcal{G}}{\partial E^{2}} d E^{2}+\frac{\partial \mathcal{G}}{\partial E} d^{2} E\right) \Delta \\
& +\Delta^{\dagger}\left(d g^{-1}-\frac{\partial \mathcal{G}}{\partial E} d E\right) d \Delta=0 .
\end{aligned}
$$

The second-order differential of the independent variable $g^{-1}$ is zero. We simplify this expression by using $\Delta^{\dagger}(\partial \mathcal{G} / \partial E) \Delta=-1$ and $\Delta^{\dagger}\left(\partial^{2} \mathcal{G} / \partial E^{2}\right) \Delta=2 \psi^{\dagger} \mathcal{G} \psi$ and by substituting $d \Delta$ from (5.6):

$$
\begin{aligned}
\frac{1}{2} d^{2} E & =\psi^{\dagger} \mathcal{G} \psi+\psi^{\dagger}\left(g d g^{-1}+\mathcal{G} d E\right) \mathcal{A}\left(d g^{-1} g+\mathcal{G} d E\right) \psi \\
& =-\psi^{\dagger} g^{-1} \psi+d \phi^{\dagger} \mathcal{A} d \phi
\end{aligned}
$$

where

$$
d \phi \equiv\left(d g^{-1} g+g^{-1} d E\right) \psi=\left(d g^{-1}-\Delta^{\dagger} d g^{-1} \Delta g^{-2}\right) \Delta .
$$

In Equation (5.8) we used the definition $\mathcal{A}^{-1}=g^{-1}-\mathcal{G}$ and the fact that $d g^{-1} \psi=0$ (the potential has been changed on infinite-repulsion sites where the wave function vanished). The first term in Equation (5.8) is real. The imaginary part of the energy is thus given by the second term:

$$
\frac{1}{2} \operatorname{Im} d^{2} E=\operatorname{Im} d \phi^{\dagger} \mathcal{A}\left(E \pm i 0^{+}, \mathbf{P}\right) d \phi,
$$

\footnotetext{
${ }^{4}$ This relation can also be proved along the lines of Appendix D. For higher derivatives, however, one will have to use the values of the Green's function on the entire lattice. Therefore, this type of analysis only works through second order.
} 
We now derive an expression for the mass of a resonance state. The problem of a resonance formed by two fermions on the same site has been addressed in an earlier work by Friedberg, Lee, and Ren [9]. They found that, as the lifetime of such a resonance increases, its mass grows without limit. This can be understood as follows. Two fermions constituting a long-lived resonance must be located on the same site. However, the motion of such a boson is mediated by consecutive hopping of the fermions to an adjacent site. This means that the fermions must part and then reunite: the boson decays into two fermions and then reappears at an adjacent site. As the decay amplitude vanishes, so does the kinetic energy of the resonance, thus giving an infinite mass. The same problem exists in the case of bound states [4. The situation changes if we consider a resonance formed by fermions on two adjacent sites. Now a pair of particles can move without changing the distance between them. On a triangular or face-centered cubic lattice, this can be accomplished with only nearest-neighbor hopping. On a square or simple cubic lattice, fermions must be able to hop to next-nearest-neighbor sites. Under such circumstances, even long-lived resonances will have a finite mass, which can readily be determined. In the limit of zero resonance width, the two fermions cannot penetrate the wall of infinite repulsion that holds them together. The wave function of such a state is non-zero for only a few relative separations. As was shown in Section II A, the relative motion of two particles with total Bloch momentum $\mathbf{P}$ is equivalent to the motion of a single particle on the same lattice with hopping amplitudes $t^{\prime}(\mathbf{r})=2 t(\mathbf{r}) \cos (\mathbf{P} \cdot \mathbf{r} / 2)$ in the external potential $g(\mathbf{r})$. As the total momentum departs from 0 , the variation of the hopping terms is quadratic in $\mathbf{P}$. The second derivative of the energy with respect to $\mathbf{P}$ gives the effective mass. Assuming that the wave function of the stable state $\psi(\mathbf{r})$ is non-degenerate at $\mathbf{P}=0$, we treat the variation in the kinetic energy for small $\mathbf{P}$ as a perturbation. To lowest order,

$$
E(\mathbf{P})=E(0)-\sum_{\mathbf{r}, \mathbf{r}^{\prime}} \psi^{*}\left(\mathbf{r}^{\prime}\right) t\left(\mathbf{r}-\mathbf{r}^{\prime}\right) \psi(\mathbf{r})\left[\frac{\mathbf{P} \cdot\left(\mathbf{r}-\mathbf{r}^{\prime}\right)}{2}\right]^{2},
$$

from which one obtains the mass. To this order, the mass is finite only for states with $\psi(\mathbf{r}) \neq 0$ on more than one site. When the state becomes unstable, its energy is altered. Since $\Delta(\mathbf{r})$ is an implicit function of the total Bloch momentum (through the variation of the fermion kinetic energy), Equation (5.5) contains the next-order correction to the energy. This correction is proportional to the inverse height of the repulsion walls and is therefore larger than the linewidth (5.10). Equation (5.5) determines the bosonic mass when (5.11) does not disperse with $\mathbf{P}$, as in the case of resonances formed on one single site.

\section{B. Resonance on a square lattice: an example}

Here we illustrate the results from the previous section. Consider a resonance that is formed on a square lattice by two fermions on the same site. This is possible when the hopping range is restricted to nearest neighbors (amplitude $t)$ and the inter-particle potential is large for two fermions on adjacent sites, $g_{1} \gg|t|$. The value of the potential $g_{0}$ on the same site controls the energy of the resonance and therefore should be within the continuum of two free fermions: $-8|t|<g_{0}<8|t|$.

We start with a stable state, which is formed in the limit of $g_{1} \rightarrow \infty$. In this case, the bound state has the wave function $\psi(\mathbf{r})=\delta_{\mathbf{r} 0}$ and the energy $E=g_{0}$. The value of $\Delta(\mathbf{r}) \equiv g(\mathbf{r}) \psi(\mathbf{r})$ on sites with an infinite repulsion is then calculated by standard perturbation methods (with (2.7) as a perturbation Hamiltonian):

$$
\Delta(\mathbf{r})=\lim _{g_{1} \rightarrow \infty} g_{1} \psi(\mathbf{r})=-2 t \cos (\mathbf{P} \cdot \mathbf{r} / 2) .
$$

We then have a $5 \times 1$ column vector $\Delta$,

$$
\Delta \equiv\left(\begin{array}{c}
\Delta(0,0) \\
\Delta(1,0) \\
\Delta(0,1) \\
\Delta(-1,0) \\
\Delta(0,-1)
\end{array}\right)=\left(\begin{array}{c}
g_{0} \\
-2 t \cos \left(P_{x} a / 2\right) \\
-2 t \cos \left(P_{y} a / 2\right) \\
-2 t \cos \left(P_{x} a / 2\right) \\
-2 t \cos \left(P_{y} a / 2\right)
\end{array}\right) .
$$

For spin-singlet states, we only need that part which is symmetric under inversion of the relative separation $\mathbf{r}$. Performing a unitary transformation, we obtain a $3 \times 1$ column vector for singlet states,

$$
\Delta=\left(\begin{array}{rr}
g_{0} & u_{0}(\mathbf{P}) \\
-2 t & u_{1}(\mathbf{P}) \\
-2 t & u_{2}(\mathbf{P})
\end{array}\right)
$$


where

$$
\begin{gathered}
u_{0}(\mathbf{P})=1, \\
u_{1}(\mathbf{P})=\cos \left(P_{x} a / 2\right)+\cos \left(P_{y} a / 2\right), \\
u_{2}(\mathbf{P})=\cos \left(P_{x} a / 2\right)-\cos \left(P_{y} a / 2\right) .
\end{gathered}
$$

In the limit as $g_{1} \rightarrow \infty$, the matrix $g^{-1}$ is given by

$$
g^{-1}=\left(\begin{array}{ccc}
g_{0}^{-1} & 0 & 0 \\
0 & 0 & 0 \\
0 & 0 & 0
\end{array}\right) .
$$

A large but finite repulsion for nearest neighbors gives

$$
d g^{-1}=\left(\begin{array}{ccc}
0 & 0 & 0 \\
0 & g_{1}^{-1} & 0 \\
0 & 0 & g_{1}^{-1}
\end{array}\right) .
$$

We then obtain the following expression for the energy of a resonance through the first order in $t / g_{1}$ :

$$
\begin{aligned}
E(\mathbf{P}) & =E^{(0)}-\Delta^{\dagger} d g^{-1} \Delta \\
& =g_{0}-\frac{4 t^{2}}{g_{1}}\left[u_{1}^{2}(\mathbf{P} / 2)+u_{2}^{2}(\mathbf{P} / 2)\right] .
\end{aligned}
$$

This gives the inverse effective mass of the resonance boson as

$$
\frac{1}{m}=\frac{4 t^{2} a^{2}}{g_{1}}
$$

and the width of the bosonic band as

$$
\Delta E=\frac{32 t^{2}}{g_{1}}
$$

To determine the resonance width, we use Equations. (5.10) and (5.9) with

$$
d \phi=-\frac{2 t}{g_{0} g_{1}}\left(\begin{array}{c}
2 t\left[u_{1}^{2}(\mathbf{P} / 2)+u_{2}^{2}(\mathbf{P} / 2)\right] \\
g_{0} u_{1}(\mathbf{P} / 2) \\
g_{0} u_{2}(\mathbf{P} / 2)
\end{array}\right) .
$$

Evaluation of $d \phi^{\dagger} \mathcal{A} d \phi$ is complicated by the fact that its inverse, $\mathcal{A}^{-1} \equiv g^{-1}-\mathcal{G}$, has a zero eigenvalue. Indeed, it follows from Equation (2.22) that

$$
\mathcal{A}^{-1} \Delta=0 \text {. }
$$

However, by its definition, vector $d \phi$ is orthogonal to $\Delta$ and $d \phi^{\dagger} \mathcal{A} d \phi$ is not singular. The calculation simplifies considerably for zero Bloch momentum of the resonance: $u_{2}(0)=0$ and the linear space becomes two-dimensional. In this case, $d \phi$ is also an eigenvector of the matrix $\mathcal{A}^{-1}$, with eigenvalue $\lambda$. The set of four equations, Equation (5.22) and $\mathcal{A}^{-1} d \phi=\lambda d \phi$, contains three identities for the matrix $\mathcal{G}$ (see Appendix $\mathbf{Q}$ ) and determines the eigenvalue $\lambda$. Solving for $\lambda$ yields

$$
\lambda=-\left(\frac{4 t}{g_{0}}+\frac{g_{0}}{4 t}\right) \mathcal{G}_{10} .
$$

Upon substituting the expression (5.23) into Equation (5.10) and expressing $\mathcal{G}_{10}$ in terms of the simpler quantity $\mathcal{G}_{00}$, we finally obtain

$$
\operatorname{Im} E=\left(\frac{16 t^{2}}{g_{1}}\right)^{2} \frac{\operatorname{Im} \mathcal{G}_{00}}{\left|1-g \mathcal{G}_{00}\right|^{2}}
$$

where $\mathcal{G}_{00} \equiv \mathcal{G}_{00}\left(E \pm i 0^{+}, 0\right)$. We see that, for both choices of the sign, the position of the pole is shifted onto the unphysical sheet. The resonance width is second-order in $t / g_{1}$, while the width of the boson band (5.20) is first-order. Therefore, in the limit of a small $t / g_{1}$, resonance bosons can propagate during their lifetime. 


\section{CONCLUSION}

We have discussed the properties of extended two-fermion bound states and resonances on a lattice by using an idealized Hubbard-like model with finite-range interactions. This two-body problem is exactly solvable on any periodic lattice, so that all approximations are well controlled. The two-body states may be relevant to high-temperature superconductors for two reasons. First, when the size of a Cooper pair in a superconductor is comparable to the average inter-particle distance, a two-fermion picture may provide a better starting point than a Fermi-sea description. The superconducting transition would then be similar to the Bose condensation in liquid ${ }^{4}$ He. Second, any description of $d$-wave superconductivity must go beyond a zero-range interaction between fermions — such as in the BCS theory - simply because the $d$-wave order parameter vanishes for two fermions on the same site.

The symmetry of the order parameter $\Delta\left(\mathbf{r}, \mathbf{r}^{\prime}\right)$ in our picture is related to that of the ground state of a pair. This observation pinpoints the important role of the fermion kinetic energy in determining the symmetry of the energy gap. It appears to us that the $d$-wave symmetry is related to the reversed sign of the hopping amplitudes along the diagonals of $\mathrm{Cu}-\mathrm{O}$ sheets, a consequence of the $d_{x^{2}-y^{2}}$ character of doped holes on copper sites. We have demonstrated that, when the magnitude of such an amplitude is comparable to that for nearest neighbors, the ground state of two fermions will be a $d_{x^{2}-y^{2}}$ wave.

In the strong-binding case, we have shown that pairs formed by fermions on adjacent lattice sites can easily move through the lattice. Thus, the condensation temperature need not be vanishingly low in this limit. This observation resloves the large-effective-mass problem raised by Noziéres and Schmitt-Rink in this connection [4]. Our conclusions in this regard are valid for narrow two-fermion resonances as well.

For weakly bound fermions, we have found that the size of a pair having a non-trivial symmetry tends to be smaller than the size of an $s$ wave. Only in the latter case is the size determined by the depth of the bound-state energy level and given by the tunneling length $\xi_{0}=\hbar v_{F} / \Delta$. In the former case, however, a pair's size is also sensitive to a short-range cutoff, which we have identified with the range of the attractive interaction. This property has its origin in the weaker tunneling exhibited by states with higher angular momenta. It is therefore not surprizing that the cuprates, quite possibly $d$-wave superconductors, have remarkably small Cooper pairs.

\section{ACKNOWLEDGMENTS}

The authors thank Professor T. D. Lee for encouragement and discussions. This work has been supported in part by U.S. Department of Energy grants DE-FG02-92 ER40699 and DOE-91 ER40651, Task B.

\section{APPENDIX A: NEAREST-NEIGHBOR ATTRACTION AND MANY-BODY BOUND STATES}

To clarify the issue of many-body bound states raised in Section III, we consider the following simple model on a square lattice. In the limit of strong attraction the fermion kinetic energy can be neglected. The interactions are given by an infinite on-site repulsion, an attractive potential $g_{S}<0$ for nearest neighbors in the spin-singlet state, and a repulsive potential $g_{T}>0$ for nearest neighbors in the spin-triplet states. Then for two nearest neighbors, the Hamiltonian can be written as

$$
\begin{aligned}
H_{\mathrm{n} . \mathrm{n} .} & =\frac{3 g_{T}+g_{S}}{4}+\left(g_{T}-g_{S}\right)\left(\mathbf{S}_{1} \cdot \mathbf{S}_{2}\right) \\
& =\frac{g_{T}+g_{S}}{2}+\frac{g_{T}-g_{S}}{2} \mathrm{P}_{12}
\end{aligned}
$$

where $\mathbf{S}_{1}$ and $\mathbf{S}_{2}$ are fermionic spins and $\mathrm{P}_{12}$ is the exchange operator.

Three fermions on a square lattice will lower their energy to

$$
E_{2+1}=g_{S}<0
$$

by forming a pair and leaving one fermion free. Alternatively, there may be two bonds connecting one fermion with the other two. The lowest energy of this system will be given by the smallest eigenvalue of the Hamiltonian,

$$
H_{3}=\frac{g_{S}+3 g_{T}}{2}+\left(g_{T}-g_{S}\right) \mathbf{S}_{1} \cdot\left(\mathbf{S}_{2}+\mathbf{S}_{3}\right)
$$


which is

$$
E_{3}=\frac{3 g_{S}+g_{T}}{2} .
$$

The "2+1" configuration then has a lower energy if $g_{T}>-g_{S}>0$. This condition can be relaxed in a fermionic ensemble. Indeed, if there are two sets of " $2+1$ " clusters on the lattice, the two free fermions will pair and reduce the energy further. Therefore, in a large ensemble,

$$
E_{2+1}=\frac{3 g_{S}}{2},
$$

and the stability of pairs requires that $g_{T}>0$, i.e. the triplet interaction must be repulsive.

Four fermions have a larger number of states from which to choose. The paired state " $2+2$ " has the lowest energy

$$
E_{2+2}=2 g_{S} .
$$

Other possibilities include clusters of four spins having three or four bonds [Figures $3(\mathrm{a})$, (b), and (c)] with the following energies:

$$
\begin{aligned}
& E_{4}^{(a)}=\frac{(3+\sqrt{3}) g_{S}+(3-\sqrt{3}) g_{T}}{2}, \\
& E_{4}^{(b)}=2 g_{S}+g_{T}, \\
& E_{4}^{(c)}=3 g_{S}+g_{T} .
\end{aligned}
$$

A comparison between Equations (A6) and (A7a b, and c) shows that pairs will merge into squares unless there is a strong enough triplet repulsion $g_{T}$. We thus obtain a condition necessary to prevent the many-body clustering in the limit of strong attraction:

$$
g_{T}>-g_{S} .
$$

Extending the analysis in this form to larger clusters of fermions is impractical due to an exponentially growing number of possible ground states. Instead, we shall give an upper bound for the triplet repulsion that ensures the stability of pairs against the formation of many-body bound states. The idea is based on the following observation. The ground-state energy of a fermionic cluster with $N$ bonds is obtained by minimizing the expectation value of the Hamiltonian

$$
H=\sum_{\langle i j\rangle}\left\{\frac{3 g_{T}+g_{S}}{4}+\left(g_{T}-g_{S}\right)\left(\mathbf{S}_{i} \cdot \mathbf{S}_{j}\right)\right\} .
$$

Minimizing the energy of each bond separately gives a crude estimate of this energy as $N g_{S}$, obviously much lower than it is. One can do better, however, by minimizing the energies of separate small clusters of bonds (not spins). Indeed, the lowest energy of a small bond cluster can also be found by minimization over all states of the large spin system. It is clear then that such a separate minimization results in a lower energy. If the bonds are grouped in $k$ small clusters, we have

$$
H=H_{1}+H_{2}+\ldots+H_{k}
$$

and therefore

$$
\min \langle H\rangle \geq \min \left\langle H_{1}\right\rangle+\min \left\langle H_{2}\right\rangle+\ldots+\min \left\langle H_{k}\right\rangle .
$$

The equality holds if all minima are reached in the same state of the spin system. In general, however, $\left[H_{i}, H_{j}\right] \neq$ 0 (whenever clusters $i$ and $j$ have common spins) and thus the eigenstates of $H_{i}$ and $H_{j}$ do not coincide. This consideration allows one to obtain a reasonable lower bound for the ground-state energy of any cluster having an even number of bonds. It can be shown that the Hamiltonian is a sum over pairs of bonds (with the two bonds of any pair sharing a common spin). The ground-state energy of two such bonds is $E_{3}$ given above Equation (A4)]. Therefore, the energy of a cluster with $N_{b}$ bonds satisfies the inequality

$$
E_{N_{b}}>N_{b} \frac{3 g_{S}+g_{T}}{4} .
$$


In the dimerized state, $N_{s} / 2$ spin pairs will have the energy

$$
E_{N_{s}}=N_{s} \frac{g_{S}}{2}
$$

which should be lower than $E_{N_{b}}$ to prevent the many-body formation. Replacing $E_{N_{b}}$ by its lower bound A12, we find that

$$
g_{T}>-g_{S}\left(3-\frac{2 N_{s}}{N_{b}}\right)
$$

is sufficient for the stability of pairs. The lowest possible ratio for $N_{s} / N_{b}$ on a square lattice is $1 / 2$, achieved when all sites on a large lattice are occupied by spins. Therefore,

$$
g_{T}>-2 g_{S} .
$$

provides a sufficient condition for a square lattice.

One can see, however, that this condition can be relaxed. As has been mentioned, the largest number of bonds originating from one spin is achieved when all spins have four nearest neighbors. In this situation, one improves the lower bound for the energy by dividing the system into clusters with four bonds originating from one spin [Figure 3(d)]. Thus, (A15) can be replaced by a milder condition.

A cluster with an odd number of bonds can be divided into a cluster with an even number of bonds and another one with three bonds. Then one can readily verify that condition (A15) again ensures the stability of pairs.

\section{APPENDIX B: POINT GROUPS OF SQUARE AND CUBIC LATTICES}

The point group of a square lattice consists of eight elements: a unit element; three proper rotations about the center of the square $(90,180,270$ degrees); two reflections with respect to the coordinate axes that are parallel to the square's sides and that have the square's center as the origin; two reflections with respect to the two diagonals of the square. These eight elements are isomorphic to the group of matrices $\pm I_{2}, \pm \tau_{1}, \pm i \tau_{2}$ and $\pm \tau_{3}$ with $I_{2}$ the $2 \times 2$ unit matrix and $\tau_{j}$ the Pauli matrices. There are five conjugate classes, which implies the following five irreducible representations: a unit representation $A_{1}$; three non-trivial one-dimensional representations $A_{2}, B_{1}$, and $B_{2}$, implemented by the functions $x_{1} y_{2}-x_{2} y_{1}, x^{2}-y^{2}$, and $x y$, respectively; a two-dimensional representation $E$, transforming as the pair of functions $(x, y)$.

The proper cubic group consists of 24 elements. In addition to the unit element, there are three $C_{4}$ axes running through the centers of opposite faces of the cube (9 rotations); four $C_{3}$ axes along the diagonals ( 8 rotations); and $6 C_{2}$ axes connecting opposite edges (6 rotations). These elements form five conjugate classes, which implies the existance of five irreducible representations: two one-dimensional representations $A_{1}$ and $A_{2}$, implemented by the

functions 1 and $x y z$; a two-dimensional representation $E$ transforming as the doublet $\left(x^{2}-y^{2}, 2 z^{2}-x^{2}-y^{2}\right)$; and two three-dimensional representations $T_{1}$ and $T_{2}$, implemented by the triplets $(x, y, z)$ and $(y z, z x, x y)$.

\section{APPENDIX C: THE GREEN'S FUNCTION AT P $=0$ FOR A SQUARE LATTICE}

The two-particle Green's function was introduced in Section II B. At a total Bloch momentum $\mathbf{P}=0$,

$$
G\left(E ; \mathbf{r}, \mathbf{r}^{\prime}\right) \equiv G\left(E, 0 ; \mathbf{r}, \mathbf{r}^{\prime}\right)=\frac{1}{\mathcal{N}} \sum_{\mathbf{q}} \frac{\psi(\mathbf{r} \mid \mathbf{q}) \psi^{*}\left(\mathbf{r}^{\prime} \mid \mathbf{q}\right)}{E-E_{\mathbf{q}}},
$$

where $\psi(\mathbf{r} \mid \mathbf{q})=\sqrt{2} \cos (\mathbf{q} \cdot \mathbf{r})$ for $\mathbf{r} \neq 0, \psi(0 \mid \mathbf{q})=1$, and $E_{\mathbf{q}}$ is the kinetic energy of two fermions with Bloch momenta $\mathbf{q}$ and $-\mathbf{q}$,

$$
E_{\mathbf{q}}=2 \sum_{\mathbf{r}} t(\mathbf{r}) e^{i \mathbf{q} \cdot \mathbf{r}}
$$

Introduce 


$$
\psi(E ; \mathbf{r})=\frac{1}{\mathcal{N}} \sum_{\mathbf{q}} \frac{e^{i \mathbf{q} \cdot \mathbf{r}}}{E-E_{\mathbf{q}}}
$$

which is the Green's function for unsymmetrized states, with $\psi(\mathbf{r} \mid \mathbf{q})=e^{i \mathbf{q} \cdot \mathbf{r}}$. If $\mathbf{r} \neq 0$ and $\mathbf{r}^{\prime} \neq 0$,

$$
\begin{aligned}
G\left(E ; \mathbf{r}, \mathbf{r}^{\prime}\right) & =\psi\left(E ; \mathbf{r}-\mathbf{r}^{\prime}\right)+\psi\left(E ; \mathbf{r}+\mathbf{r}^{\prime}\right), \\
G(E ; \mathbf{r}, 0) & =G(E ; 0, \mathbf{r})=\sqrt{2} \psi(E ; \mathbf{r}), \\
G(E ; 0,0) & =\psi(E ; 0) .
\end{aligned}
$$

It is straightforward to verify that $\psi(E ; \mathbf{r})$ satisfies the equation

$$
E \psi(E ; \mathbf{r})-2 \sum_{\mathbf{r}^{\prime}} t\left(\mathbf{r}^{\prime}\right) \psi\left(E ; \mathbf{r}+\mathbf{r}^{\prime}\right)=\delta_{\mathbf{r}, 0}
$$

We shall restrict the hopping range to nearest neighbors (amplitude $t_{1}$ ) and next-nearest neighbors (amplitude $t_{2}$ ). Then the kinetic energy of two fermions is

$$
E_{\mathbf{q}}=4 t_{1}\left(\cos q_{x} a+\cos q_{y} a\right)+8 t_{2} \cos q_{x} a \cos q_{y} a
$$

The minimum of $E_{\mathbf{q}}$ appears at $\mathbf{q}=0$ when both $t_{1}<0$ and $t_{1}+2 t_{2}<0$. As has been shown in section II $\mathrm{C}$, the ground-state wave function of two fermions has an alternating sign only if there exist positive hopping amplitudes. We therefore consider the case $t_{2}>0$, which may be relevant to cuprate superconductors.

At $\mathbf{P}=0$, the two-fermion energy band lies between $E_{\min }=E_{(0,0)}=8 t_{1}+8 t_{2}$ and $E_{\max }=E_{(\pi, \pi)}=-8 t_{1}+8 t_{2}$. The density of states diverges when the line of constant $E$ touches the boundary of the Brillouin zone, at $E_{\mathrm{vH}}=$ $E_{(\pi, 0)}=-8 t_{2}$. It will be convenient to measure the energy from the van Hove singularity:

$$
\begin{aligned}
E^{\prime} & =E+8 t_{2}, \\
E_{\min }^{\prime} & =8 t_{1}+16 t_{2} \leq 0, \\
E_{\max }^{\prime} & =-8 t_{1}+16 t_{2}>0 .
\end{aligned}
$$

On an infinite lattice, the Green's function for relative separation $\mathbf{r}=(m, n)$ is

$$
\begin{aligned}
& \psi(m, n)= \\
& \int \frac{d^{2} \mathbf{q}}{(2 \pi)^{2}} \frac{\cos m q_{x} \cos n q_{y}}{E-4 t_{1}\left(\cos q_{x}+\cos q_{y}\right)-8 t_{2} \cos q_{x} \cos q_{y}}
\end{aligned}
$$

When $n=0$, the integration over $q_{y}$ is particularly simple. We have

$$
\begin{aligned}
& \psi(m, 0)= \\
& \frac{1}{\pi} \int_{0}^{\pi} \frac{\cos m q_{x} d q_{x}}{\sqrt{\left[E^{\prime}-E_{\min }^{\prime} \cos ^{2}\left(q_{x} / 2\right)\right]\left[E^{\prime}-E_{\max }^{\prime} \sin ^{2}\left(q_{x} / 2\right)\right]}} .
\end{aligned}
$$

The sign of the square root is determined as follows. When the root is real, it has the same sign as $E^{\prime}$, which ensures that $\psi(E ; 0,0) \approx 1 / E$ as $E \rightarrow \infty$. The region for imaginary values of the square root, between the branch points $E_{\min }^{\prime} \sin ^{2}\left(q_{x} / 2\right)$ and $E_{\max }^{\prime} \cos ^{2}\left(q_{x} / 2\right)$, is accessed either from $E^{\prime}=E_{\max }^{\prime} \cos ^{2}\left(q_{x} / 2\right)+0^{+}>0$ (by encircling the singularity counterclockwise), or from $E^{\prime}=E_{\min }^{\prime} \sin ^{2}\left(q_{x} / 2\right)-0^{+}<0$ (by encircling the singularity clockwise). In both cases, the square root has a positive imaginary part and zero real part.

At the origin, $\psi$ can be expressed in terms of a complete elliptic integral of the first kind,

$$
\psi(0,0)=\frac{2}{\pi E^{\prime}} \mathbf{K}(k)
$$

where

$$
k^{2}=1-\left(1-E_{\min }^{\prime} / E^{\prime}\right)\left(1-E_{\max }^{\prime} / E^{\prime}\right) .
$$

This function can be analytically continued into the region $E_{\min }^{\prime}<E^{\prime}<E_{\max }^{\prime}$ using the identity 


$$
\mathbf{K}(k)=\frac{\mathbf{K}(1 / k) \pm i \mathbf{K}^{\prime}(1 / k)}{k}
$$

(the sign is chosen in accordance with the rule stated above). The density of states is then given by

$$
\mathcal{D}(E)=-\frac{\operatorname{Im} G(0,0)}{\pi}=\frac{2}{\pi^{2}\left|E^{\prime}\right| k} \mathbf{K}^{\prime}(1 / k) .
$$

This function is plotted in Figure 1 for $t_{1}=-1$ and several positive values of $t_{2}$. As $t_{2} \rightarrow-t_{1} / 2$, the band flattens at the bottom and the logarithmic van Hove singularity coalesces into the branch point of the Green's function. This strongly enhances the density of states near the lower edge of the band.

As $m$ increases, expressions for $\psi(E ; m, 0)$ become progressively more complicated and include, in general, complete elliptic integrals of all three kinds. For example,

$$
\begin{aligned}
\psi(1,0)= & \frac{4}{\pi}\left(\frac{1}{2 E^{\prime}}-\frac{1}{E_{\max }^{\prime}}\right) \mathbf{K}(k) \\
& -\frac{4}{\pi}\left(\frac{1}{E^{\prime}}-\frac{1}{E_{\max }^{\prime}}\right) \Pi\left(E^{\prime} / E_{\max }^{\prime}, k\right),
\end{aligned}
$$

where the integral of the third kind is defined as

$$
\Pi\left(\alpha^{2}, k\right)=\int_{0}^{1} \frac{d x}{\left(1-\alpha^{2} x^{2}\right) \sqrt{\left(1-x^{2}\right)\left(1-k^{2} x^{2}\right)}} .
$$

An expression like (C18) provides little insight into the behavior of the Green's function. Fortunately, it is possible to obtain an approximation for $\psi(E ; \mathbf{r})$ in the vicinity of its singular points. Several authors [10] have identified the enhanced density of states near the van Hove singularity as a possible candidate for explaining high critical temperatures in cuprate superconductors. In view of that, we discuss the leading behavior of $\psi(E ; \mathbf{r})$ near $E=E_{\mathrm{vH}}$, i.e. $E^{\prime}=0$.

As $E^{\prime} \rightarrow 0, k \rightarrow \infty$, so that $\mathbf{K}(1 / k) \rightarrow \pi / 2$ and $\mathbf{K}^{\prime}(1 / k)-\ln 4 k \rightarrow 0$. Therefore, near $E^{\prime}=0$,

$$
\psi(0,0) \approx \frac{1}{E_{0}^{\prime}}-\frac{2 i}{\pi E_{0}^{\prime}} \log \frac{4 E_{0}^{\prime}}{E^{\prime}} .
$$

Note that $\operatorname{Re} \psi(0,0) \rightarrow \pm 1 / E_{0}^{\prime}$ as $E^{\prime} \rightarrow 0^{ \pm}$, in accordance with the sign rule for the square root. At even sites along the axes, the difference $\psi(2 m, 0)-\psi(0,0)$ remains finite as $E^{\prime} \rightarrow 0$ :

$$
\begin{aligned}
\psi(2 m, 0)-\psi(0,0) & \rightarrow \frac{2 i}{\pi E_{0}^{\prime}} \int_{0}^{\pi} d q_{x} \frac{1-\cos 2 m q_{x}}{\sin q_{x}} \\
& =\frac{8 i}{\pi E_{0}^{\prime}} \sum_{j=1}^{m} \frac{1}{2 j-1} .
\end{aligned}
$$

In a similar way,

$$
\psi(2 m+1,0)-\psi(1,0) \rightarrow 0 .
$$

It is also seen from (C13) that the two divergent contributions to $\psi(2 m+1,0)$, near $q_{x}=0$ and $q_{x}=\pi$, have opposite signs and thus tend to cancel each other. In the limit as $E^{\prime} \rightarrow 0$,

$$
\psi(1,0) \rightarrow \frac{1}{E_{0}^{\prime}}-\frac{i}{\pi E_{0}^{\prime}} \log \frac{E_{\max }^{\prime}}{-E_{\min }^{\prime}} .
$$

This shows that $\psi(2 m+1,0)$ is not singular at $E=E_{\mathrm{vH}}$.

Using Equation (C7) it is now possible to obtain approximations for $\psi(\mathbf{r})$ on all sites. We demonstrate how this is done for the singular part of the Green's function. As $E \rightarrow E_{\mathrm{vH}}=-8 t_{2}$, Equation (C7) reads

$$
-8 t_{2} \psi(\mathbf{r})-2 t_{1} \sum_{\text {n.n. }} \psi\left(\mathbf{r}^{\prime}\right)-2 t_{2} \sum_{\text {n.n.n. }} \psi\left(\mathbf{r}^{\prime \prime}\right)=\mathcal{O}(1),
$$


where $\mathbf{r}^{\prime}$ refers to the four nearest neighbors of $\mathbf{r}$, while $\mathbf{r}^{\prime \prime}$ refers to the four next nearest sites. Starting with $\mathbf{r}=0$, we obtain (by using symmetry of the lattice)

$$
\psi(1,1)=-\psi(0,0)+\mathcal{O}(1) .
$$

Applying Equation (C24) to site $(1,0)$, we find that

$$
\psi(2,1)=\mathcal{O}(1),
$$

because the divergent contributions of sites $(0,0)$ and $(2,0)$ are cancelled by those of $(1,1)$ and $(1,-1)$. We now separate the lattice into two sublattices, putting adjacent sites on different sublattices. In general, $\psi(\mathbf{r})$ is singular on the sublattice that contains the origin: at sites with two even coordinates, $\psi(\mathbf{r})=\psi(0,0)+\mathcal{O}(1)$; at sites with two odd coordinates, $\psi(\mathbf{r})=-\psi(0,0)+\mathcal{O}(1)$. On the other sublattice, $\psi(\mathbf{r})$ is regular at $E=E_{\mathrm{vH}}$. This means that the relative motion of the particles at $E=E_{\mathrm{vH}}$ is restricted to one of the sublattices.

As we mentioned, on any site $\mathbf{r}, \psi(\mathbf{r})$ is a linear combination of three complete elliptic integral with modulus (C15). Therefore, it should be possible to find $\psi(\mathbf{r})$ on the whole lattice if it is known on three conveniently chosen sites. For example, one starts out with $(0,0),(1,0)$, and $(2,0)$. Then $\psi(1,1)$ is found from the Laplace equation (C7) around site $(0,0)$ by using the symmetry arguments $\psi(m, n)=\psi(n, m)$ and $\psi(-m, n)=\psi(m, n)$. The Laplace equation on site $(1,0)$ expresses $\psi(2,1)$ in terms of $\psi$ at sites $(0,0),(1,0),(1,1)$, and $(2,0)$. To proceed, we use an idea due to Friedberg and Martin [11], who showed that there is a lattice analog of the gradient condition, $\nabla \psi(\mathbf{r}) \| \mathbf{r}$. Extending their idea to the case of several hopping amplitudes, we find an additional relation between values of $\psi\left(\mathbf{r}+\mathbf{r}^{\prime}\right)$ on sites surrounding $\mathbf{r}$.

$$
\sum_{\mathbf{r}^{\prime}}^{\prime} \frac{t\left(\mathbf{r}^{\prime}\right)}{x^{\prime}} \psi\left(\mathbf{r}+\mathbf{r}^{\prime}\right)=\sum_{\mathbf{r}^{\prime}}^{\prime} \frac{t\left(\mathbf{r}^{\prime}\right)}{y^{\prime}} \psi\left(\mathbf{r}+\mathbf{r}^{\prime}\right)
$$

where $\sum^{\prime}$ means that sites with $x^{\prime}=0\left(y^{\prime}=0\right)$ are excluded. On sites with high symmetry, such as $(m, m),(m, 0)$, and $(0, m)$, this equation becomes an identity and supplies no additional information.

Now we are in a position to recover $\psi$ on the remainder of the lattice. We set up the Laplace equations on sites $(2,0)$ and $(2,1)$ in order to relate $\psi$ at sites $(3,0),(3,1)$, and $(3,2)$ to each other and to $\psi$ at sites where it has been calculated. The gradient equation on site $(2,1)$ becomes a third one in this set of linear equations, which yields three more sites. $\psi(3,3)$ is obtained from the Laplace equation on site $(2,2)$. This establishes the algorithm for computing $\psi$ on the remaining sites. When $\psi$ is known on the square with the corners $(0,0),(0, m),(m, 0)$, and $(m, m)$, we write the Laplace equation for site $(m, 0)$ and the Laplace and gradient equations for site $(m, 1)$. We then solve these equations for $\psi$ at $(m+1,0),(m+1,1)$, and $(m+1,2)$. Using the Laplace equations for sites $(m, 2), \ldots,(m, m)$, we move up and complete the side of the square.

In two particular cases, $t_{2}=0$ and $2 t_{2}=-t_{1}$, the calculation of $\psi(\mathbf{r})$ is simplified by the absence of elliptic integrals of the third kind. In the latter case, the van Hove singularity coalesces with the bottom of the continuum band, $E_{\min }^{\prime}=0$, resulting in a stronger divergence of $\psi(E ; \mathbf{r})$ near $E^{\prime}=0$. We write down an approximate expression for $\psi(E, \mathbf{r})$ without presenting a derivation. Just outside the continuum band, as $E^{\prime} \rightarrow 0^{-}$,

$$
\begin{aligned}
& \psi(m, n) \sim-\frac{4|m n|}{E_{\max }^{\prime}}-\frac{1}{\pi \sqrt{-E^{\prime} E_{\max }^{\prime}}} \\
& \times\left[\log \left(-\frac{16 E_{\max }^{\prime}}{E^{\prime}}\right)-4 \sum_{k=1}^{m} \frac{1}{2 k-1}-4 \sum_{l=1}^{n} \frac{1}{2 l-1}\right],
\end{aligned}
$$

which can be readily continued to the region $0<E^{\prime}<E_{\max }^{\prime}$; the point $E^{\prime}=0$ should be encircled clockwise.

\section{APPENDIX D: PROOF OF IDENTITIES USED IN SECTION V}

To prove the relation

$$
-\Delta^{\dagger} \frac{\partial \mathcal{G}}{\partial E} \Delta=1
$$

we write the quantity $-\Delta^{\dagger}(\partial \mathcal{G} / \partial E) \Delta \equiv \delta$ in its explicit form, 


$$
\delta=-\sum_{i, j=0}^{n} \psi^{*}\left(\mathbf{R}_{i}\right) g\left(\mathbf{R}_{i}\right) \frac{\partial G\left(\mathbf{R}_{i}, \mathbf{R}_{j}\right)}{\partial E} g\left(\mathbf{R}_{j}\right) \psi\left(\mathbf{R}_{j}\right) .
$$

It follows then from the definition of the Green's function (2.21) and the orthogonality relation (2.14) for functions $\psi(\mathbf{r} \mid \mathbf{p})$ that

$$
\frac{\partial G\left(\mathbf{r}^{\prime}, \mathbf{r}\right)}{\partial E}=-\sum_{\mathbf{r}^{\prime \prime}} G\left(\mathbf{r}^{\prime}, \mathbf{r}^{\prime \prime}\right) G\left(\mathbf{r}^{\prime \prime}, \mathbf{r}^{\prime}\right)
$$

The sum must be taken over the entire lattice, so that this identity cannot be applied directly to the $n \times n$ matrix $\mathcal{G}(E, \mathbf{P})$. We substitute this expression into (D2a):

$$
\begin{aligned}
\delta & =\sum_{i, j=0}^{n} \sum_{\mathbf{r}} \psi^{*}\left(\mathbf{R}_{i}\right) g\left(\mathbf{R}_{i}\right) G\left(\mathbf{R}_{i}, \mathbf{r}\right) G\left(\mathbf{r}, \mathbf{R}_{j}\right) g\left(\mathbf{R}_{j}\right) \psi\left(\mathbf{R}_{j}\right) \\
& =\sum_{\mathbf{r}, \mathbf{r}^{\prime}, \mathbf{r}^{\prime \prime}} \psi^{*}\left(\mathbf{r}^{\prime \prime}\right) g\left(\mathbf{r}^{\prime \prime}\right) G\left(\mathbf{r}^{\prime \prime}, \mathbf{r}\right) G\left(\mathbf{r}, \mathbf{r}^{\prime}\right) g\left(\mathbf{r}^{\prime}\right) \psi\left(\mathbf{r}^{\prime}\right) \\
& =\sum_{\mathbf{r}} \psi^{*}(\mathbf{r}) \psi(\mathbf{r})=1 .
\end{aligned}
$$

In $(\mathrm{D} 2 \mathrm{~b})$, the newly added terms do not contribute because either $\psi\left(\mathbf{r}^{\prime}\right)=0$ or $g\left(\mathbf{r}^{\prime}\right)=0$ for sites $\mathbf{r}^{\prime} \neq \mathbf{R}_{j}$. The transition from (D2b) to (D2d) is justified by Equations (5.1a) and (5.1b).

Next, we prove that, in $\mathcal{A}(\partial \mathcal{G} / \partial E) \Delta, \partial \mathcal{G} / \partial E$ can be replaced by $-\mathcal{G}^{2}$. Explicitly,

$$
\begin{aligned}
& \sum_{j, k=0}^{n} A\left(\mathbf{R}_{i}, \mathbf{R}_{j}\right) \frac{\partial G\left(\mathbf{R}_{j}, \mathbf{R}_{k}\right)}{\partial E} g\left(\mathbf{R}_{k}\right) \psi\left(\mathbf{R}_{k}\right) \\
= & -\sum_{j, k=0}^{n} \sum_{\mathbf{r}} A\left(\mathbf{R}_{i}, \mathbf{R}_{j}\right) G\left(\mathbf{R}_{j}, \mathbf{r}\right) G\left(\mathbf{r}, \mathbf{R}_{k}\right) g\left(\mathbf{R}_{k}\right) \psi\left(\mathbf{R}_{k}\right) \\
= & -\sum_{j=0}^{n} \sum_{\mathbf{r}} A\left(\mathbf{R}_{i}, \mathbf{R}_{j}\right) G\left(\mathbf{R}_{j}, \mathbf{r}\right) \psi(\mathbf{r}) \\
= & -\sum_{j, k=0}^{n} A\left(\mathbf{R}_{i}, \mathbf{R}_{j}\right) G\left(\mathbf{R}_{j}, \mathbf{R}_{k}\right) \psi\left(\mathbf{R}_{k}\right) \equiv-\mathcal{A G} \psi
\end{aligned}
$$

[1] Z. X. Shen et al., Phys. Rev. Lett. 70, 1553 (1993); J. Chem. Phys. Solids 54, 1169 (1993).

[2] C. C. Tsuei et al., Phys. Rev. Lett. 73, 593 (1994); J. R. Kirtley et al., Nature 373, 225 (1995).

[3] P. Monthoux, A. Balatsky, and D. Pines, Phys. Rev. B 46, 961 (1992); P. Monthoux and D. Pines, Phys. Rev. B 47, 6069 (1993); ibid., 49, 4261 (1994).

[4] P. Nozières and S. Schmitt-Rink, J. Low Temp. Phys. 59, 195 (1985).

[5] R. Friedberg and T. D. Lee, Phys. Lett. A 138, 423 (1989); Phys. Rev. B 40, 6745 (1989).

[6] R. Micnas, J. Ranninger, and S. Robaszkiewicz, Rev. Mod. Phys. 62, 113 (1990), and references therein.

[7] R. Friedberg, T. D. Lee, and H. C. Ren, Ann. Phys. 208, 149 (1991).

[8] A. P. Mackenzie et al., Phys. Rev. Lett. 71, 1238 (1993); M. S. Osofsky et al., ibid., 2315 (1993).

[9] R. Friedberg, T. D. Lee, and H. C. Ren, Phys. Rev. B 50, 10190 (1994).

[10] See, e.g., E. Dagotto, A. Nazarenko, and A. Moreo, Phys. Rev. Lett. 74310 (1995); C. C. Tsuei, in Proceedings of CCAST Symposium on High- $T_{c}$ superconductivity and the $C_{60}$ family, Beijing, China, May 1994, ed. by S. Feng and H. C. Ren (Gordon and Breach, 1995).

[11] R. Friedberg and O. Martin, J. Phys. A 20, 5095 (1990). 


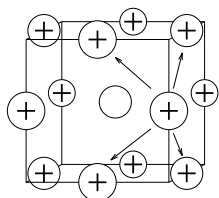

(a)

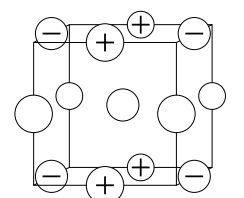

(b)

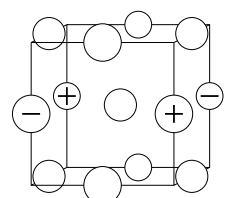

(c)

FIG. 1. Deep bound states on an f.c.c. lattice. Symmetries and energies of the states: (a) $A_{1}, g_{1}+8 t$. (b) $E$, $g_{1}-4 t$. (c) $T_{2}, g_{1}$.

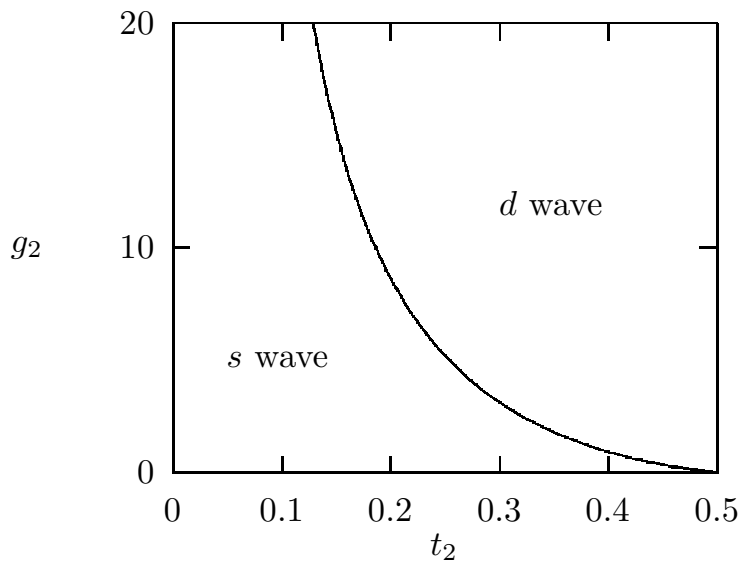

FIG. 2. Shallow $s$-wave and $d$-wave ground states on a square lattice. Nearest-neighbor hopping $t_{1}=-1$.

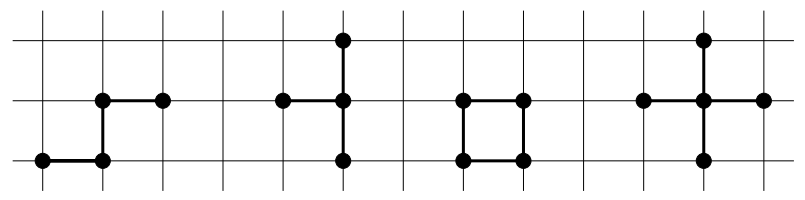

(a)

(b)

(c)

(d)

FIG. 3. Bond clusters on a square lattice.

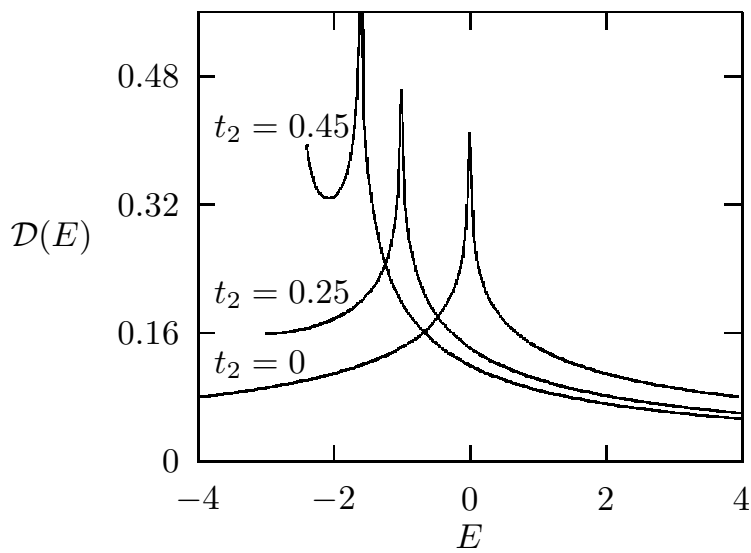

FIG. 4. Density of states for different values of nearest-neighbor hopping $t_{2} \cdot t_{1}=-1$. Values of $t_{2}$ are shown on the graph. 